\title{
The Reaction ${ }^{7} \operatorname{Li}\left(\pi^{+}, \pi^{-}\right)^{7} \mathbf{B}$ and its Implications for ${ }^{7} \mathbf{B}$ *
}

\author{
J. Pätzold, R. Bilger, H. Clement, K. Föhl ${ }^{\dagger}$, J. Gräter, \\ R. Meier, D. Schapler ${ }^{\ddagger}$ and G.J. Wagner \\ Physikalisches Institut der Universität Tübingen, \\ Auf der Morgenstelle 14, D-72076 Tübingen \\ A. Denig and W. Kluge \\ Institut für Experimentelle Kernphysik, Universität Karlsruhe \\ M. Schepkin \\ Institute for Theoretical and Experimental Physics, Moscow
}

\begin{abstract}
The reaction ${ }^{7} \operatorname{Li}\left(\pi^{+}, \pi^{-}\right)^{7} \mathrm{~B}$ has been measured at incident pion energies of 30-90 MeV. ${ }^{7} \mathrm{Li}$ constitutes the lightest target nucleus, where the pionic charge exchange may proceed as a binary reaction to a discrete final state. Like in the $\Delta$ resonance region the observed cross sections are much smaller than expected from the systematics found for heavier nuclei. In analogy to the neutron halo case of ${ }^{11} \mathrm{Li}$ this cross section suppression is interpreted as evidence for a proton halo in the particle-unstable nucleus ${ }^{7} \mathrm{~B}$.
\end{abstract}

PACS numbers: 25.80.Gn, 27.20.+n, 14.20.Pt

In recent years the pionic double charge exchange (DCX) reaction has received much attention at energies below the $\Delta$ resonance, mainly for two reasons. On the one hand the cross sections there have been found to be sensitive to nucleon-nucleon (NN) correlations of short range, a feature which has been looked for since long in this genuine $2 \mathrm{~N}$ reaction [1]. On the other hand the forward-angle cross sections exhibit an unexpected though systematic resonance-like energy dependence with peak cross sections between $T_{\pi}=45$ and 70 $\mathrm{MeV}$, which in general are substantially larger than those in the $\Delta$-resonance region and above. Combination of both these features has led to the so-called $d^{\prime}$ hypothesis [2,3], which postulates the existence of a NN-decoupled $\pi \mathrm{NN}$ resonance with $I\left(J^{P}\right)=$ even $\left(0^{-}\right), m \approx 2.06 \mathrm{GeV}$ and vacuum width $\Gamma_{\pi \mathrm{NN}} \approx 0.5 \mathrm{MeV}$ to explain the observed effects in DCX. In the nuclear medium the width of such a resonance is broadened very much by "Fermi smearing" due to the motion of the nucleon pair active in the DCX process as well as by collision damping due to $d^{\prime} N \rightarrow 3 N$, so that the resulting width in the nuclear medium lies in the order of 20 to $30 \mathrm{MeV}$. The question whether this picture is correct or whether a subtle, as of yet not understood medium effect is the origin of

\footnotetext{
*supported by the BMBF (06 TU 886), DFG (Mu 705/3, Graduiertenkolleg), NFR and INTAS RFBR (95-605)

${ }^{\dagger}$ Present address: Department of Physics and Astronomy, University of Edinburgh

${ }^{\ddagger}$ Present address: SAP, Walldorf, Germany
}

this resonance-like structure, is not easy to settle within the DCX, since the free process on a dinucleon is not observable. However, one could expect to minimize the influence of such contingent medium effects by studying the DCX process on the lightest nuclei possible. ${ }^{7} \mathrm{Li}$ is the lightest nucleus where this reaction may still proceed to a discrete final nuclear state, though the ${ }^{7} \mathrm{~B}$ ground state is already $3.65 \mathrm{MeV}$ above the proton emission threshold. As a result it has a natural width of $1.4(2) \mathrm{MeV}$ [4] corresponding to a lifetime of $5 \times 10^{-22} \mathrm{~s}$. This is still in the order of magnitude of the classical orbiting time for the 3 valence protons outside the alpha core in ${ }^{7} \mathrm{~B}$. Hence it appears justified to ask about the radius of these proton orbits, in particular whether they possibly form a proton halo. A comparable case has recently been discussed regarding proton-unstable states in ${ }^{17} \mathrm{~F}$ 汭. With regard to DCX a similar situation on the neutron side has recently been met in the ${ }^{11} \mathrm{~B}\left(\pi^{-}, \pi^{+}\right)^{11} \mathrm{Li}$ reaction, where it has been demonstrated [6] that this reaction is extremely sensitive to the neutron halo in ${ }^{11} \mathrm{Li}$ and provides a reliable determination of its radius.

The measurements have been carried out with the LEPS magnetic spectrometer [7] at the $\pi \mathrm{E} 3$ channel at PSI. Sheets of metallic Lithium with an areal density of $265 \mathrm{mg} / \mathrm{cm}^{2}$ and a ${ }^{7} \mathrm{Li}$ isotopic purity of about $95 \%$ served as target. Absolute cross sections have been obtained by use of the lepton normalization method [7] which is based on the measurement of elastic $\mu$ scattering. The simultaneously measured elastic $\pi$ scattering serves as cross check for the validity of this method. The cross sections obtained this way for elastic $\pi$ scattering from ${ }^{7} \mathrm{Li}$ are in very good agreement with optical model calculations using the $\mathrm{J} 4$ potential [8], which is known to provide reliable predictions for low pion energies. Sample spectra obtained for the DCX reaction at $\Theta_{l a b}=30^{\circ}$ are shown in Fig. 11 for low and high incident energies. In both spectra the peak corresponding to the transition to the ${ }^{7} \mathrm{~B}$ ground state (GST) is clearly visible, however, the continuum due to the breakup channels ${ }^{6} \mathrm{Be}+\mathrm{p}$ and ${ }^{4} \mathrm{He}+3 \mathrm{p}$ with Q-values of +2.28 and $+3.65 \mathrm{MeV}$, respectively, relative to the ${ }^{7} \mathrm{~B}$ ground state increases strongly with increasing incident energy. Hence especially for the higher energies a proper treatment of the continuum is important for a reliable extraction of the peak content. In ref. [9] it has been shown that the continuum due to breakup of light nuclei is not well described by pure 
phase space, in particular at its high-energy end, where the breakup fragments have very low relative energies and hence are likely to undergo substantial final state interactions (FSI). Therefore we have taken into account FSI [10] in the calculation of the shape of the breakup continuum. It is significant particularly for the two-body breakup ${ }^{6} \mathrm{Be}+\mathrm{p}$ which starts with a steep rise right at the position of the GST peak. Fig. 1 shows the decomposition of the experimental data into continuum part (dashed lines) and GST peak. The description of the continuum part is accomplished by adjusting the absolute magnitude of the calculated distributions for the two breakup channels (dotted) to the data. The GST peak is described by a Gaussian the width of which has been calculated from the experimental resolution obtained in the corresponding elastic scattering runs.

The experimental angular distributions for the GST are shown in Fig. 2 for the different incident energies. The error bars include the uncertainties both from statistics and from the decomposition of the spectra into GST peak and breakup continuum. The observed angular dependence is rather weak as expected for monopole transitions on light nuclei. This is also borne out by the calculations (dashed curves, adjusted in height to the data) performed within the $d^{\prime}$ model, which will be discussed below in more detail. Since in this model the angular dependence is governed by the c.m. motion of the active nucleon pair in initial and final nuclear states, i.e. by the two-nucleon form factor, the predicted angular dependence equals essentially the one expected from a standard DCX treatment. The data are compatible with the predicted angular dependence up to $T_{\pi} \approx 60 \mathrm{MeV}$. For the higher energies $T_{\pi} \gtrsim 70 \mathrm{MeV}$, however, where the calculations predict an increasing fall-off towards larger angles, the data exhibit a nearly opposite behavior with cross sections tending to increase with increasing angle. This change in the experimental angular behavior is clearly borne out by comparing the $55 \mathrm{MeV}$ and $70 \mathrm{MeV}$ data sets. Since at high incident energies the GST peaks sit upon a large continuum of breakup channels, the question might arise whether some incorrect treatment of the latter might have affected the GST data. However, we tried various descriptions of the background and none led to significant changes in the GST angular dependence. If there is no experimental problem with the data at these higher energies, then this change in the angle dependence has to be associated with some physical origin. As will be discussed below, beyond $60 \mathrm{MeV}$ the $d^{\prime}$ amplitude gets already small compared to the conventional amplitude, which we identify with the tail of the $\Delta \Delta$ process [1], so that the angular distributions no longer need to be governed by the $d^{\prime}$ mechanism. We only note in passing that in the DCX on still lighter nuclei, the He isotopes, the angular distributions for incident energies between 70 and $120 \mathrm{MeV}$ are observed to be practically isotropic, too 11,12 .

Fig. 3 displays the energy dependence of the forward angle cross section. For the $\Delta$ resonance region there exists one measurement at $T_{\pi}=180 \mathrm{MeV}$ and $\Theta_{l a b}=5^{\circ}$ [13]. In order to compare with its result we have ex- trapolated our data to $\Theta_{l a b}=5^{\circ}$ by the curves shown in Fig. 2. Since the angular distributions are very flat these extrapolations are quite moderate except at the highest energies. Since there the observed angular dependence is at variance with the $d^{\prime}$ predictions we give two values in Fig. 3 for $T_{\pi} \geq 65 \mathrm{MeV}$, the one obtained with the $d^{\prime}$ angular dependence as for the lower energies (crosses), and the one obtained simply by assuming an isotropic angular dependence as suggested by the data (dots).

The most conspicuous and common feature of both the LAMPF datum and our measurements is the surprisingly small cross sections, which are much smaller than expected from systematics. For the discussion of this issue we first turn to the $\Delta$ resonance region, where a great deal of systematic DCX studies have been undertaken. There both analog and nonanalog transitions have been shown [1.14 to exhibit very simple systematic dependences on the target mass A, which may be understood in simple diffractive models of the DCX process in the $\Delta$ region. Fig. 1 shows as an example the forward angle data 13 15 for the nonanalog GSTs at $T_{\pi}=164$ and $180 \mathrm{MeV}$, respectively. At both energies the data are in excellent agreement with the expected $A^{-4 / 3}$ dependence with the exception of two cases, the ${ }^{11} \mathrm{~B}\left(\pi^{-}, \pi^{+}\right)^{11} \mathrm{Li}$ cross section 115$]$ at $164 \mathrm{MeV}$ and the ${ }^{7} \mathrm{Li}\left(\pi^{+}, \pi^{-}\right)^{7} \mathrm{~B}$ cross section [13] at $180 \mathrm{MeV}$. Both are roughly a factor of three below their value expected from the $A^{-4 / 3}$ systematics. For the ${ }^{11} \mathrm{~B}$ case this huge discrepancy has recently been successfully explained by the neutron halo of ${ }^{11} \mathrm{Li}$. According to Gibbs and Hayes [6] GSTs are very sensitive to a change of the active nucleons' orbital radius in the transition from initial to final states. In the limiting case of extreme halo radii the cross section is expected to scale as the inverse sixth power of the halo radius. For realistic cases this dependence is much more moderate. In the case of ${ }^{11} \mathrm{~B}\left(\pi^{-}, \pi^{+}\right){ }^{11} \mathrm{Li}$ the ratio of the measured cross section $\sigma$ over the value $\sigma_{0}$ expected from systematics is $1 / 3.3(4)$. This ratio has been related in ref. [6] to a change of the radius of the active proton pair $R_{2 p}$ in ${ }^{11} \mathrm{~B}$ to that of the active neutron pair $R_{2 n}$ in ${ }^{11} \mathrm{Li}$ by $R_{2 n} / R_{2 p}=1.9(3)$. This represents rather a quadratic dependence. The latter uncertainty includes also systematic uncertainties in the calculations (wave functions, distortion etc.). The resulting halo radius of $R_{2 n}=5 \mathrm{fm}$ for ${ }^{11} \mathrm{Li}$ determined this way agrees very well with that obtained by other means [15].

It is therefore very tempting to try to explain the surprisingly low cross sections for ${ }^{7} \operatorname{Li}\left(\pi^{+}, \pi^{-}\right)^{7} \mathrm{~B}$ in full analogy to the above example. In this case we would rather deal with a proton halo in ${ }^{7} \mathrm{~B}$, which appears to be not unplausible in view of the particle-unbound character of this nucleus, as discussed already in the introduction. From Fig. A we see that in this case the suppression is $\sigma_{0} / \sigma=2.9(4)$, which translates into $R_{2 p} / R_{2 n}=1.6(3)$, if we transfer the findings for ${ }^{11} \mathrm{Li}$ (Fig. 1 of ref. [6]) straight to our case. However, we have to be a bit more careful, since in contrast to ${ }^{11} \mathrm{~B}$ the target nucleus ${ }^{7} \mathrm{Li}$ has already an unusually large charge radius due to its loosely bound $\alpha+t$ cluster structure. With $2.4 \mathrm{fm}$ its root-mean-square radius is roughly $15 \%$ larger than ex- 
pected from the systematics of nuclear radii. Since the $A^{-4 / 3}$ dependence of forward angle cross sections derived in diffractive models results from geometrical considerations and represents essentially a $R^{-4}$ dependence, the increased ${ }^{7} \mathrm{Li}$ radius causes $\sigma_{0}$ already to be lower by a factor of about 1.7. This leads then to a reduced suppression of $\sigma_{0} / \sigma=1.7(2)$, which translates into a value of $R_{2 p} / R_{2 n}=1.3(2)$ by Fig. 1 of ref. [6].

As pointed out in ref. [6] the reduction factor in cross section due to the change of $R_{2 N}$ is largely independent of the reaction mechanism and results primarily from the overlap of the initial and final NN-wave functions. Hence also the $d^{\prime}$ model, which contains this overlap, too, and which we will use for the description of our lowenergy data, should be adequate for estimating $R_{2 p} / R_{2 n}$, even from the datum at $T_{\pi}=180 \mathrm{MeV}$. The function $\sigma_{0} / \sigma=f\left(R_{2 p} / R_{2 n}\right)$ calculated this way is shown as inset in Fig. 3, it yields for the ratio of $\sigma_{0} / \sigma=1.7(2)$ a value of $R_{2 p} / R_{2 n}=1.2-1.3$ which agrees very well with the value obtained above.

With this in mind we now turn to the discussion of the low-energy data. The first striking feature there is that the peak cross section is much below the cross section at the $\Delta$ resonance, a feature observed so far in no other case. Even for the neighboring cases ${ }^{12} \mathrm{C}$ and ${ }^{16} \mathrm{O}$ [3] the low-energy peak cross sections are still well above those in the $\Delta$-resonance region. Since the low-energy cross sections are known to depend strongly on NN correlations, it is tempting to seek the solution there. Indeed, the unusually large charge radius of ${ }^{7} \mathrm{Li}$ leads to a considerable reduction of the probability to find the valence neutrons active in the DCX process at small relative distances. In the $d^{\prime}$ calculations this alone gives a reduction by roughly a factor of two in cross section compared to the case, where the usual $A^{1 / 3}$ dependence for the radius of ${ }^{7} \mathrm{Li}$ is assumed. Another strong reduction in the $d^{\prime}$ cross section results, if in addition for ${ }^{7} \mathrm{~B}$ a proton halo is assumed as discussed in the preceding section. The cross sections calculated this way are displayed in Fig. 3. The dotted curve shows the result, if we use $R_{2 p} / R_{2 n}=1.3$, as derived from the $180 \mathrm{MeV}$ datum, and a value of $\Gamma_{\text {spread }}=10 \mathrm{MeV}$ for the collision damping. These calculations account already quite well for the data, only the calculated resonance structure appears to be somewhat too narrow. This can be improved if we use $\Gamma_{\text {spread }}=15 \mathrm{MeV}$ instead, but then the calculated peak cross section gets somewhat too low. The calculations may be brought back to the data if we readjust the radius ratio to $R_{2 p} / R_{2 n}=1.2$ (solid curve in Fig. 3). So our conclusions about a proton halo in ${ }^{7} \mathrm{~B}$ are somewhat dependent on $\Gamma_{\text {spread }}$. The data clearly prefer the larger value for $\Gamma_{\text {spread }}$ and hence the more moderate value for the change in radii. Yet, the value $R_{2 p} / R_{2 n}=1.2$ is still in good agreement with the one derived above from the cross section in the $\Delta$-resonance region.

In conclusion, the measured cross sections for the DCX on ${ }^{7} \mathrm{Li}$ leading to the ground state in ${ }^{7} \mathrm{~B}$ are much smaller than expected from systematics both in the $\Delta$ resonance region and below. This suppression can partly be attributed to the exceptionally large radius of ${ }^{7} \mathrm{Li}$ and partly to an even larger proton halo in ${ }^{7} \mathrm{~B}$ with a radius of about $3 \mathrm{fm}$, i.e. as big as the radius of nuclei in the Ca region. The low-energy data show again some peak structure in the energy dependence, though much less pronounced than observed for other nuclei. Within the $d^{\prime}$ model this suppression is understood as being due to the low NN separation densities at short relative distances because of the large radii in ${ }^{7} \mathrm{Li}$ and in particular in ${ }^{7} \mathrm{~B}$. The observed angular dependence is well understood at energies, where the $d^{\prime}$ amplitude is the dominating process. At higher energies, where the $d^{\prime}$ amplitude is small compared to other processes, the experimental angular distributions get surprisingly flat, a phenomenon not yet understood.

[1] for a survey see, e.g. M.B. Johnson and C.L. Morris, Ann. Rev. Nucl. Part. Sci. 43 (1993) 165; H. Clement, Prog. Part. Nucl. Phys. 29 (1992) 175; and references therein

[2] R. Bilger, H.A. Clement and M.G. Schepkin, Phys. Rev. Lett. 71 (1993) 42 and 72 (1994) 2972

[3] K. Föhl et al., Phys. Rev. Lett. 79 (1997) 3849

[4] see F. Ajzenberg-Selove, Nucl. Phys. A490 (1988) 1

[5] R. Morlock et al., Phys. Rev. Lett. 79 (1997) 3837

[6] W.R. Gibbs and A.C. Hayes, Phys. Rev. Lett. 67 (1991) 1395

[7] B.M. Barnett et al., Nucl. Instr. Meth. A297 (1990) 444; H. Matthäy et al., Proc. Int. Symp. on Dynamics of Collective Phenomena in Nuclear and Subnuclear Long Range Interactions in Nuclei, Bad Honnef 1987 (ed. P. David) World Scientific 1988, 542

[8] O. Meirav et al., Phys. Rev. C40 (1989) 843

[9] K.K. Seth, Int. Workshop on Pions in Nuclei, Penyscola 1991 (eds. E. Oset, M.J. Vicente-Vacas, C. Garcia Recio) World Scientific 1992, 205

[10] see, e.g. M.L. Goldberger and K.M. Watson, Collision Theory (John Wiley \& Sons, New York, 1964); M. Schepkin, O. Zaboronsky, H. Clement, Z. Phys. A345 (1993) 407

[11] M. Yuly et al., Phys. Rev. C55 (1997) 1848

[12] J. Gräter et al., Phys. Lett. B420 (1998) 37 and Phys. Rev. C, accepted for publication

[13] K.K. Seth, Second LAMPF Int. Workshop on PionNucleus Double Charge Exchange, Los Alamos 1989 (eds. W.R. Gibbs and M.J. Leitch) World Scientific 1990, 473

[14] R. Gilman et al., Phys. Rev. C35 (1987) 1334 and references therein

[15] T. Kobayashi, Nucl. Phys. A538 (1992) 343c and A553 (1993) $465 \mathrm{c}$ 


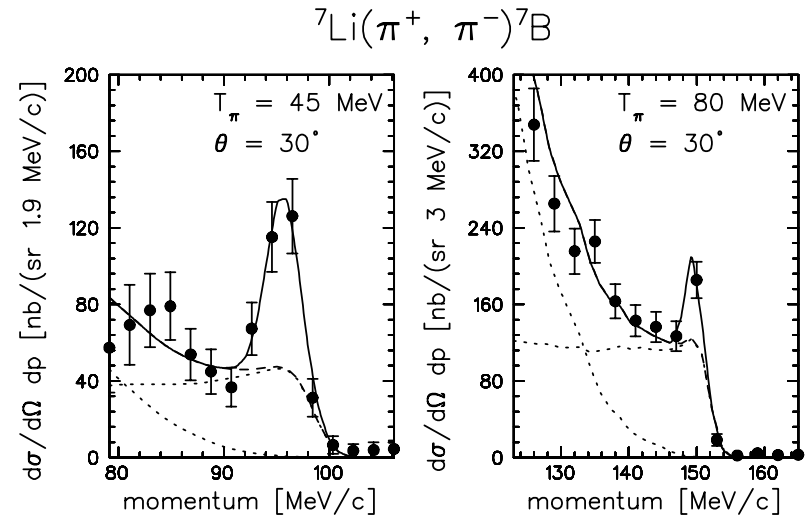

FIG. 1. Sample DCX spectra taken at $T_{\pi}=45$ and 80 $\mathrm{MeV}$, respectively, at a scattering angle of $\Theta_{l a b}=30^{\circ}$. The dotted lines represent ${ }^{6} \mathrm{Be}+\mathrm{p}$ and ${ }^{4} \mathrm{He}+3 \mathrm{p}$ breakup channels. The transition to the ${ }^{7} \mathrm{~B}$ ground state is fitted by a Gaussian, the width of which has been determined from the experimental resolution measured in corresponding elastic scattering runs.

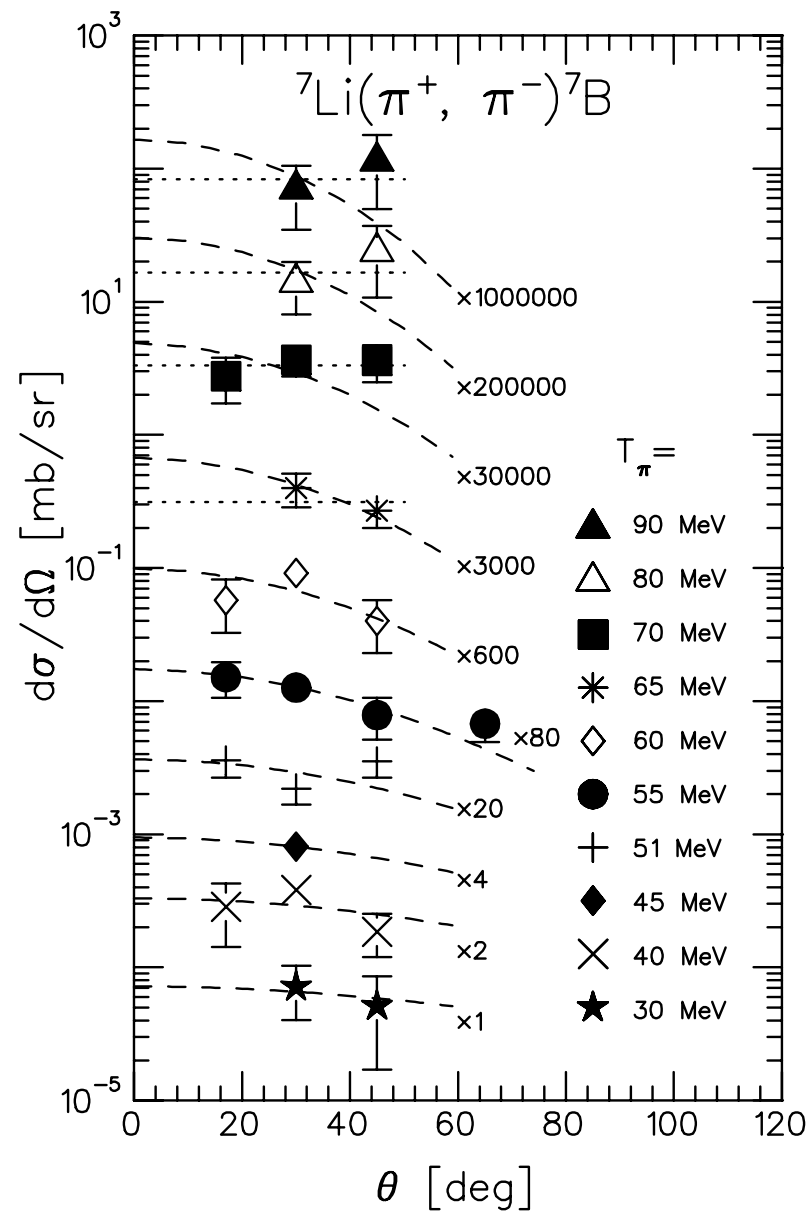

FIG. 2. Angular distributions of the GST in the energy range $T_{\pi}=30-90 \mathrm{MeV}$. The dashed curves represent $d^{\prime}$ calculations fitted in height to the data. The horizontal lines characterize an isotropic angular dependence fitted to the data for $T_{\pi} \geq 65 \mathrm{MeV}$.

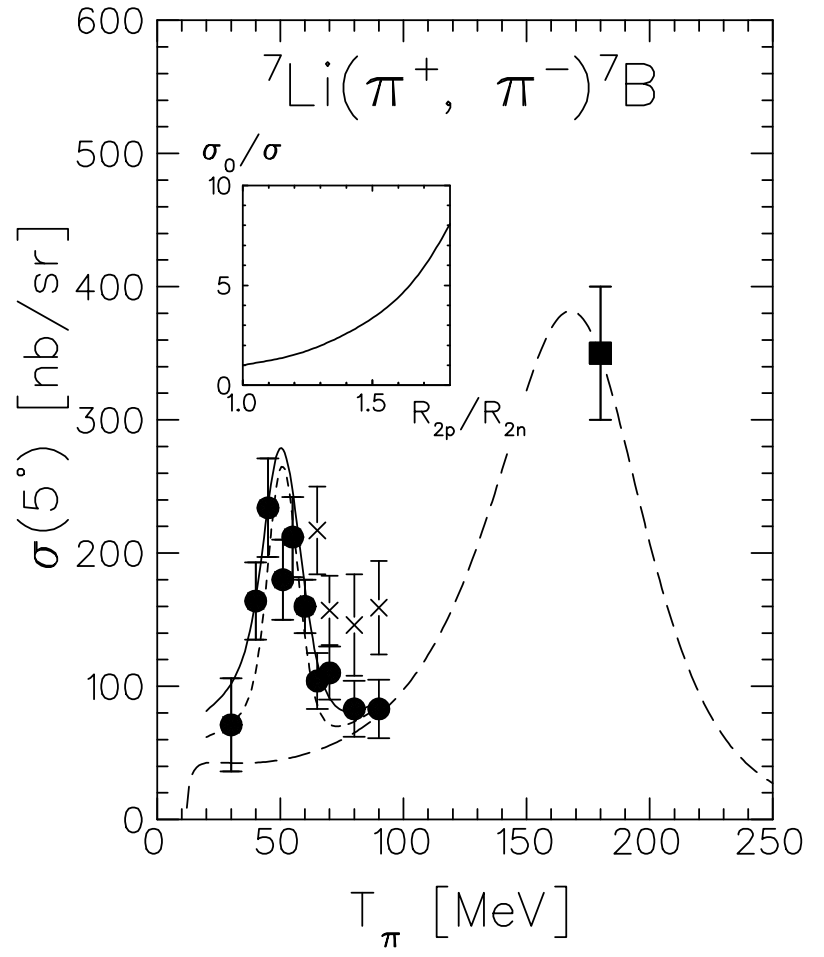

FIG. 3. Energy dependence of the forward angle cross section $\sigma\left(\Theta=5^{\circ}\right)$. The data point at $T_{\pi}=180 \mathrm{MeV}$ is from ref. [13]. At low energies our extrapolated values as obtained from Fig. 2 are shown. For $T_{\pi} \geq 65 \mathrm{MeV}$ we give two values: for the solid dots an isotropic angular distribution is assumed as suggested by the data, for the crosses the validity of the $d^{\prime}$ angular distributions is assumed though they miss the trend in the data. The dashed lines represent the $\Delta \Delta$ process in a phenomenological parametrization. The dotted and solid curves give the result, when the $d^{\prime}$ amplitude is added coherently using $\Gamma_{\text {spread }}=10$ and $15 \mathrm{MeV}$, respectively. The inset shows the change in the calculated cross section due to a change in the orbit radius of the active NN pair (see text).

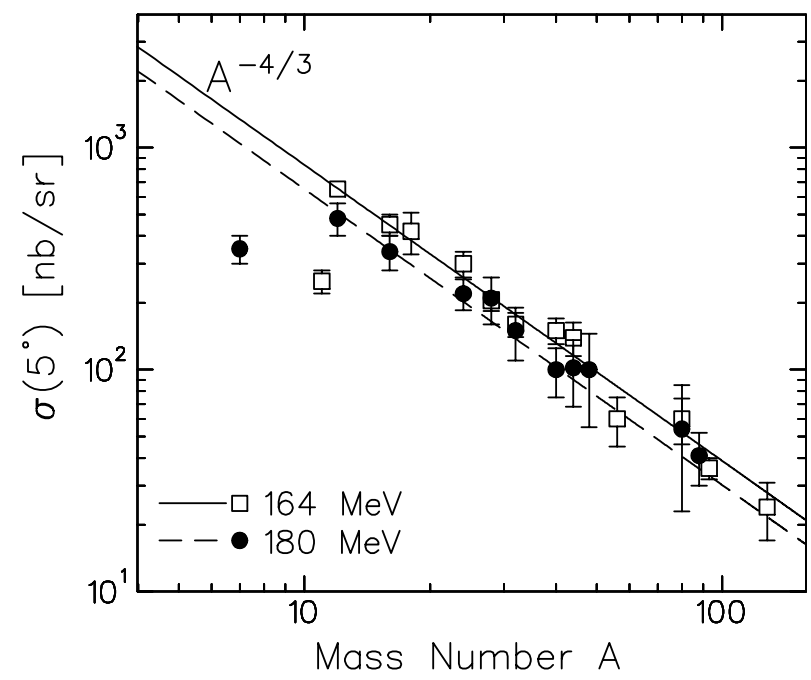

FIG. 4. Systematics of the GSTs in the $\Delta$-resonance region. Open (solid) symbols represent the data at $T_{\pi}=164(180)$ $\mathrm{MeV}$ (from refs. 13 15). The solid and dashed lines give the $A^{-4 / 3}$ dependence fitted to these data. 